\title{
THE INFLUENCE OF CONDUCTOR PARTICLE SIZE DISTRIBUTION ON THE BLENDING CURVE OF EPOXY-BASED THICK FILM RESISTORS
}

\author{
BULENT ULUG, JOHN M. ROBERTSON and PETER J.S. EWEN ${ }^{\dagger}$ \\ Department of Electrical Engineering, University of Edinburgh, Edinburgh, UK.
}

(In final form March 24, 1982)

The influence of conductor particle size distribution on the blending curve of epoxy-based thick film resistors has been investigated. It was found that the critical volume fraction shifts to higher values as the spread of the conductor particle size distribution decreases. The results are interpreted in terms of a model whose main parameter is the contact probability for the conductor particles.

\section{INTRODUCTION}

There has been growing interest in epoxy-based thick film resistors (TFR) systems because they offer advantages of low cost and low temperature processing. However, the conduction mechanism in this type of system is complex and differs from that in cermet TFR's. ${ }^{1-3}$ In particular, because the insulating phase in epoxy-based TFR pastes is not particulate as it is in the case of cermet TFR pastes, film performance is critically dependent on the size distribution of the conducting particles.

The effect of particle size distribution on the electrical characteristics of any class of TFR has not been studied in much depth and most published results have concentrated on the temperature characteristics of cermet TFR's. ${ }^{4,5}$ Present theories dealing with the conductivity of metal insulator composites ${ }^{6-9}$ do not take into account the size distribution of the conducting particles and so are not applicable to epoxy-based TFR's. In addition, a number of these theories ${ }^{8,9}$ have been developed for systems made from powder mixtures and are not valid for the case of conducting particles dispersed at random in a continuous insulating medium. Classical percolation theory not appropriate in the case of epoxy-based resistors, ${ }^{1}$ although it has been successfully applied to a variety of composite systems ${ }^{10}$ including cermet TFR's. ${ }^{11}$ An empirical expression for the blending curve of epoxy-based TFR's has been derived by Shen- $\mathrm{Li}^{1}$ but as he does not ascribe any physical meaning to the parameters used, the expression does not provide much insight into the conduction mechanism in these materials.

An alternative approach based on contact probability of conducting particles has been used in this paper since it is a physically realistic description of epoxy-based TFR's and the contact probability in the mixture can be adjusted by controlling the volume fraction and particle size distribution of the conducting phase.

\section{DEVELOPMENT OF A THEORETICAL RELATIONSHIP}

The usual practice in calculating the blending curve of thick film resistors is to calculate the film resistivity as a function of conductor volume fraction and the resistivity of the constituents. The alternative approach presented in this paper, however, concerns

\footnotetext{
†Present address: Physics Dept., University of Edinburgh, Edinburgh, UK.
} 
the critical volume fraction. This approach allows the use of a relatively simple technique, since unlike the film resistivity, the critical volume fraction is basically determined by a small number of factors. (i.e. the structure of the constituents ${ }^{8,11}$ and the lattice type). ${ }^{12}$

The first step in the development of a model is to calculate the contact probability, $\mathrm{P}_{c}$, for the whole film rather than for a particular conductive path. It is assumed that the conductive particles are spherical in shape and are distributed to form a simple cubic structure so that the particle-to-particle distance, $\mathrm{L}$, can be written as

$L=\left(\frac{V}{n_{T}}\right)^{1 / 3}=V_{c}\left[\int_{V_{\min }}^{V_{\max }} \frac{f\left(v_{i}\right)}{v_{i}} d v_{i}\right]^{-1 / 3}$

where $\mathrm{V}$ is the total volume, $\mathrm{n}_{\mathrm{T}}$ is the number of particles in the film, $\mathrm{V}_{\mathrm{c}}$ is the conductor volume fraction, $\mathrm{V}_{\min }$ and $\mathrm{V}_{\max }$ are the volume of the smallest and largest particles respectively and $f\left(v_{i}\right)$ is the probability of finding a particle with volume $v_{i}$.

In the system described above, two particles with radius $r_{i}$ and $r_{j}$ will be in contact if $L \lesssim r_{i}+r_{j}$ or, in terms of $r_{i}$, if

$$
r_{j} \gtrsim L-r_{i}
$$

Although only the nearest neighbours can satisfy Equation 2, there exists a finite, nonzero probability

$$
\mathrm{J}=\mathrm{f}\left(\mathrm{r}_{\mathrm{i}}\right) \times \mathrm{f}\left(\mathrm{r}_{\mathrm{k}}\right) \quad \mathrm{r}_{\min } \lesssim_{\mathrm{r}_{\mathrm{k}}} \lesssim_{\mathrm{r}_{\max }}
$$

that the kth particle could also be near the ith particle. Here $f\left(r_{i}\right)$ is the probability of finding a particle with radius $r_{i}$ in the film and $r_{\min }$ and $r_{\max }$ are the radius of the smallest and largest particles respectively. In this way, a degree of randomness is introduced into the system. For a uniform particle size distribution, $f\left(r_{j}\right)=\delta\left(r_{i}-r\right)$ where $\delta\left(r_{i}-r\right)$ is the Dirac delta function and $r$ is the radius of the particles; randomness is not important since the individual particles cannot be distinguished. In fact, in this case $J$ is always equal to 1 and Equation 2 determines whether all or none of the particles in the system are in contact.

However, for a system having a non-uniform particle size distribution both Equation 2 and Equation 3 can only describe individual pairs. In this case, Equation 3 can be considered as the contact probability of two particles provided that $r_{k}$ satisfies Equation 2 . Such a system can then be conveniently described by a contact probability which may be defined as the ratio of the probability sums of the potential contacts, $I_{c}$, to all possible combinations, $I\left(P_{c}=I_{c} / I\right)$. Since the actual number of particles which satisfy Equation 2 is limited. (Figure 1), the probability sum of the potential contacts, $I_{c}$, will always be smaller than I.

From Equation 2, Equation 3 and Figure 1, $I_{c}$ can be calculated. To avoid counting pairs of particles twice, the integral limits have been adjusted and the result is given by

$I_{c}=\int_{r_{\min }}^{L / 2} \int_{L-r_{i}}^{r_{\max }} f\left(r_{j}\right) f\left(r_{j}\right) d r_{i} d r_{j}+\int_{L / 2}^{r_{\max }} \int_{r_{i}}^{r_{\max }} f\left(r_{j}\right) f\left(r_{j}\right) d r_{i} d r_{j}$

The probability sum of all possible combinations can be defined as $I=I_{c}+I_{n c}$ where $I_{n c}$ is the probability sum of contacts that are not possible and given by 


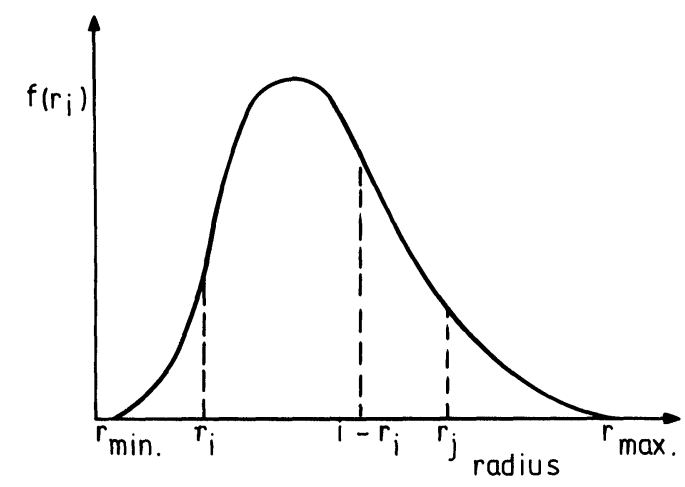

FIGURE 1 Probability distribution of particle radius in a many-particle system.

$I_{n c}=\int_{r_{\text {min }}}^{L / 2} \int_{r_{i}}^{L-r_{i}} f\left(r_{j}\right) f\left(r_{j}\right) d r_{i} d r_{j}$

For $\mathrm{f}\left(\mathrm{v}_{\mathbf{i}}\right)=$ constant, and thus $\mathrm{f}\left(\mathrm{r}_{\mathbf{i}}\right)=$ constant, from the normalized condition

$\int_{0}^{\infty} f\left(v_{i}\right) d v_{i}=1$

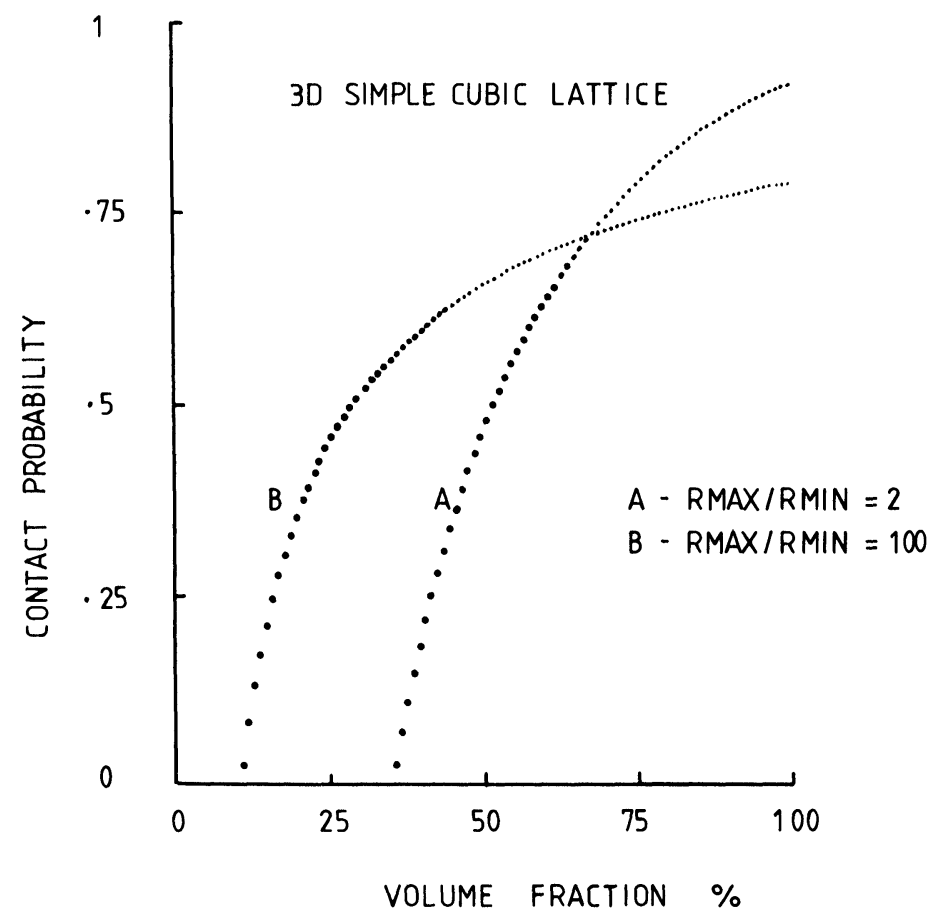

FIGURE 2 Contact probability vs. conductor volume fraction for two $X$ values and $f\left(v_{i}\right)=$ consts. in $3 \mathrm{D}$. 
it is found that $f\left(V_{\mathfrak{i}}\right)=1 /\left(V_{\max }-V_{\text {min }}\right)$ and from Equation $1 \mathrm{~L}$ is given by

$L=\left(\frac{4 \pi}{9}\right)^{1 / 3} r_{\min }\left(\frac{X^{3}-1}{\ln X}\right)^{1 / 3} \frac{1}{V_{c}^{1 / 3}}$

Here $X=r_{\max } / r_{\min }$ and is a measure of the width of the particle size distribution for the particular distribution considered. The contact probability $P_{c}\left(P_{c}=I_{c} / I_{c}+I_{n c}\right)$ can then be calculated from Equation 4, Equation 5 and Equation 7 and is given by

$P_{c} \frac{(X-1)^{2}-2\left(\frac{A}{2}-1\right)^{2}}{(X-1)^{2}}$

Here $A=L / r_{\min }$ where $L$ is given by Equation 7. In Figure 2, Equation 8 is plotted against $\mathrm{V}_{\mathrm{c}}$. As might be expected, it is a continuous function of $\mathrm{V}_{\mathrm{c}}$ and increases with increasing conductor volume fraction. However, it does not show the volume fraction at which a conducting path between the two electrodes appears for the first time; this is known as the critical volume fraction (CVF).

To each CVF there corresponds a critical contact probability and this must be known in order to determine the CVF. In Figure 3, corresponding CVF's of four arbitrarily chosen critical contact probabilities are plotted against X. As can be seen the CVF is expected to shift toward zero with increasing $\mathrm{X}$ and with decreasing critical probability. These results will be reflected in the resistivity vs. conductor volume fraction graph.

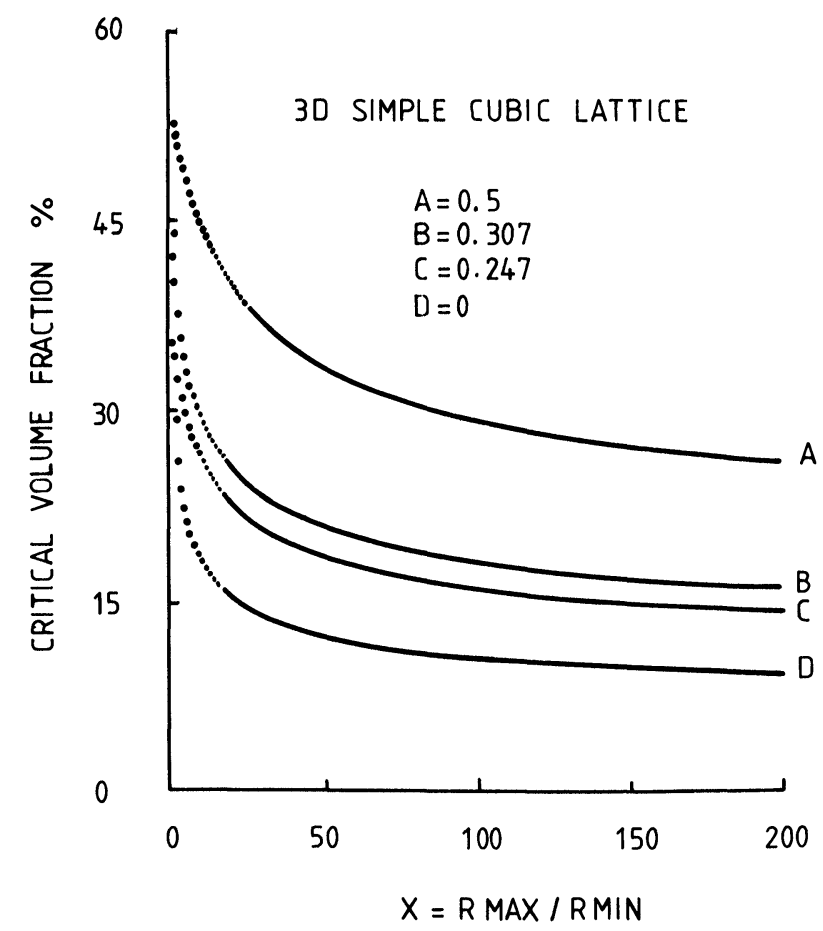

FIGURE 3 Critical volume fraction vs. $X$ for four critical contact probabilities for $f\left(v_{\mathbf{i}}\right)=$ const. in 3D. 
TABLE I

Particle size distribution data for the $\mathrm{Ag}$ and $\mathrm{C}$ powders

\begin{tabular}{lllll}
\hline Powder & $\begin{array}{l}\text { Shape of } \\
\text { Particles }\end{array}$ & $\begin{array}{l}\text { Minimum particle } \\
\text { radius } \mathrm{r}_{\min }(\mu \mathrm{m})\end{array}$ & $\begin{array}{l}\text { Maximum particle } \\
\text { radius } \mathrm{r}_{\max }(\mu \mathrm{m})\end{array}$ & $\begin{array}{l}\mathrm{X} \\
\left(\mathrm{r}_{\max } / \mathrm{r}_{\min }\right)\end{array}$ \\
\hline $\mathrm{Ag}(10)$ & Spherical & 0.5 & 25 & 50 \\
$\mathrm{Ag}(18)$ & Spherical & 0.5 & 10 & 20 \\
$\mathrm{C}(1)$ & Irregular & 0.5 & 40 & 80 \\
$\mathrm{C}(3)$ & Irregular & 0.5 & 50 & 100 \\
\hline
\end{tabular}

\section{EXPERIMENTAL RESULTS}

Resistivity measurements have been made on samples composed of epoxy resin loaded with carbon or silver-coated glass particles. Four mixtures were used and their principal features are summarised in Table 1. Data on particle size and shape were obtained from SEM photographs. Different batches of carbon provided sufficiently different particle size distributions but fractionation techniques were used to produce the distribution of finer silver particles.

The samples were prepared by first mixing equal portions of resin and hardener and then stirring in the conductive powder. In the case of Ag-based systems, alumina lids of dimensions $17 \times 5 \times 0.8 \mathrm{~mm}$ were filled with the mixtures; opposite faces of the lids were coated with ESL $9635 \mathrm{Pd} / \mathrm{Ag}$ conductive paste and fired at $850^{\circ} \mathrm{C}$ in a belt furnace prior to filling. For the carbon-based systems, plastic boxes of dimensions $27 \times 18 \times$ $14 \mathrm{~mm}$ were filled with the mixtures; rectangular stainless steel electrodes were fixed at opposite ends of the boxes prior to filling. All samples were air cured. Resistance measurements were made at least 100 hours after filling at room temperature to make sure that the polymerisation was completed. Resistance values less than $14 \mathrm{M} \Omega$ were measured with a Solatron 7065 digital voltmeter whereas high values of resistance were measured with a Keithley $610 \mathrm{C}$ electrometer. The blending curves obtained for the four systems are shown in Figure 4.
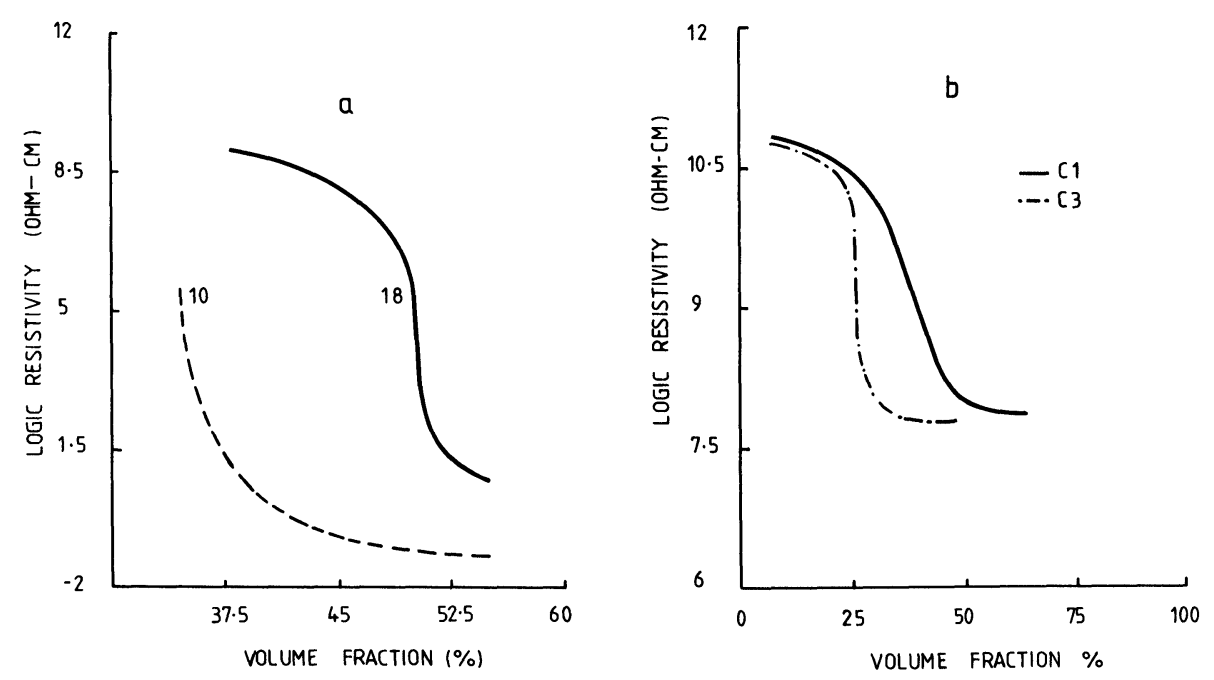

FIGURE 4 Log resistivity vs. $V_{c}$ for (a) the silver-and (b) the carbon-based systems. 


\section{DISCUSSION}

Before discussing the experimental results it should be noted that the critical contact probability has a similar physical meaning as the critical percolation probability. The critical contact probability indicates when a conducting path between the two electrodes appears for the first time. However, the region over which the transition from insulator to conductor occurs cannot be described by a single critical contact probability. For this purpose, it is convenient to define two critical contact probabilities, $P_{1}$ and $P_{h}$. The lowest critical contact probability, $\mathrm{P}_{1}$, can be interpreted as the contact probability at which an extensive network of conductive particles is actually formed for the first time. The latter, $\mathrm{p}_{\mathrm{h}}$, is identical with the critical percolation probability. ${ }^{12}$

Two critical probabilities enable us to see how the width of the transition region, $\Delta \mathrm{V}$, depends on particle size distribution. $\Delta \mathrm{V}$ is calculated from Equation 8 for four sets of arbitrarily chosen $P_{1}$ and $P_{h}$ for $f\left(v_{i}\right)=$ const. and plotted against $X$ in Fig. 5. A very sharp transition, i.e. zero width, results if the two critical probabilities are very close or equal to each other and also if the particles have a uniform size distribution $(X=1)$.

Thus the model predicts that as the particle size range broadens, i.e. as $\mathrm{X}$ increases, the CVF's shift to lower values and the width of the metal-insulator transition region decreases. It is clear from Figure 4 that the observed results for both carbon and silverbased systems qualitatively agree with these predictions. The data obtained by Shen- $\mathrm{Li}^{1}$ for polymer-based TFR's loaded with carbon black also support this model. He shows that a carbon black with large average particle size yields a lower CVF, which corresponds to $P_{h}$, and a narrower transition region than a carbon black with a small average particle size. (It is possible that for these systems the average particle size reflects the range of particle sizes). We have found no other data in the literature with which to compare our results.

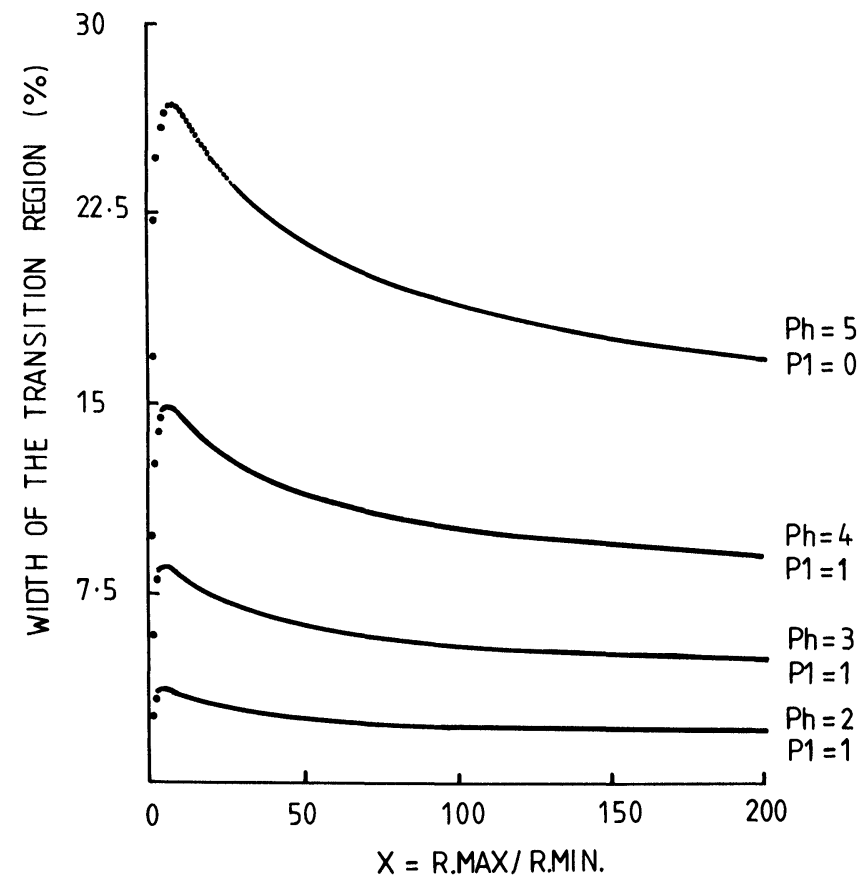

FIGURE 5 Width of the transition region vs. $X$ for $f\left(v_{i}\right)=$ Const. in 3 dimensions. The sets of critical contact probabilities were chosen arbitrarily. 
The numerical values were evaluated by using a constant volume distribution function. A more realistic distribution function would give better quantitative agreement with the experiment and would yield results which were dependent on the average particle size as well as the spread of particle size distribution. However, because of the cubic relation between volume and radius, the relation between the volume distribution function, $f\left(V_{i}\right)$, and the radius distribution function, $f\left(r_{j}\right)$, will not be linear and the analysis will become considerably more complex.

The shape of the particles has not been taken into account in the model. Kusy ${ }^{7}$ has shown that the contact probability increases with increasing irregularity of the conductive particles, which in turn decreases the CVF.

The practical implications of the model are that, first, to obtain reproducible intermediate sheet resistivities a wide transition region is necessary and hence tight control of the particle size distribution is essential. Secondly, because the thickness of the epoxylayer between the particles is nearly constant and is relatively small for values of $V_{c}$ below the CVF's for narrow size ranges, resistors with high resistivity and small TCR values can be prepared by controlling the particle size distribution and choosing the correct average particle size.

\section{CONCLUSIONS}

A model has been proposed to account for the effect of particle size distribution on the blending curves of epoxy-based TFR systems. The model is based on the contact probability for the conductor particles and predicts that as the particle size range broadens the CVF's shift to lower values and the width of the metal-insulator region decreases. It is shown that the sharp transition will result if the critical contact probabilities are very close to each other and if the particles have a uniform size distribution. The model has been tested with data on two types of system and is found to give agreement with experiment.

\section{ACKNOWLEDGEMENTS}

This work was supported by the Science and Engineering Research Council (SERC). One of us (BU) is grateful to the Ministry of Education of Turkey for providing a scholarship.

\section{REFERENCES}

1 Shen-Li Fu, "Studies on the sheet resistivity of polymer thick film resistors". IEEE 30th Electronic Conference 188-192 (1980).

2 F.F.T. de Araujo and H.M. Rosenberg, "Switching behaviour and DC electrical conductivity of epoxy-resin/metal-powder composites", J. Phys. D: 9 1025-2030 (1976).

3 F. Beuche, "A new class of switching materials". J. Appl. Phys., 44 532-533 (1976).

4 R.M. Hill and T.J. Coutts, "Charge transfer in discontinuous thin and cermet films". Thin Solid Films, 42 201-212 (1977).

5 A.H. Boonstra and C.A.H. Mutsaers, "The effect of particle size on the temperature coefficient of resistance of thick film resistors". Thin Solid Films, 67, 13-20 (1980).

6 R.M. Scarisbrick, "Electrically conducting mixtures", J. Phys. D: 6 2098-2110 (1973).

7 A. Kusy, "Chain of conducting particles that determine the resistivity of thick resistive films". Thin Solid Films, 43 243-250 (1977).

8 A. Malliaris and D.T. Turner, "Influence of particle size on the electrical resistivity of compacted mixtures of polymeric and metallic powders". J. Appl. Phys., 42 604-618 (1971). 
9 D.P.H. Smith and J.C. Anderson, "Electrical conduction in thick film paste resistors". Thin Solid Films, 38 313-321 (1976).

10 T.J. Coutts, "Percolation conduction in mixed insulator-conductor systems". Thin Solid Films. 38 79-89 (1980).

11 G.E. Pike, "Conductivity of thick film (cermet) resistors as a function of metallic particle volume fraction". AIP Conference. Proc. 40 366-71 (1978).

12 S. Kirkpatrik, "Percolation and Conduction". Rev. Mod. Phys., 45 574-588 (1973). 

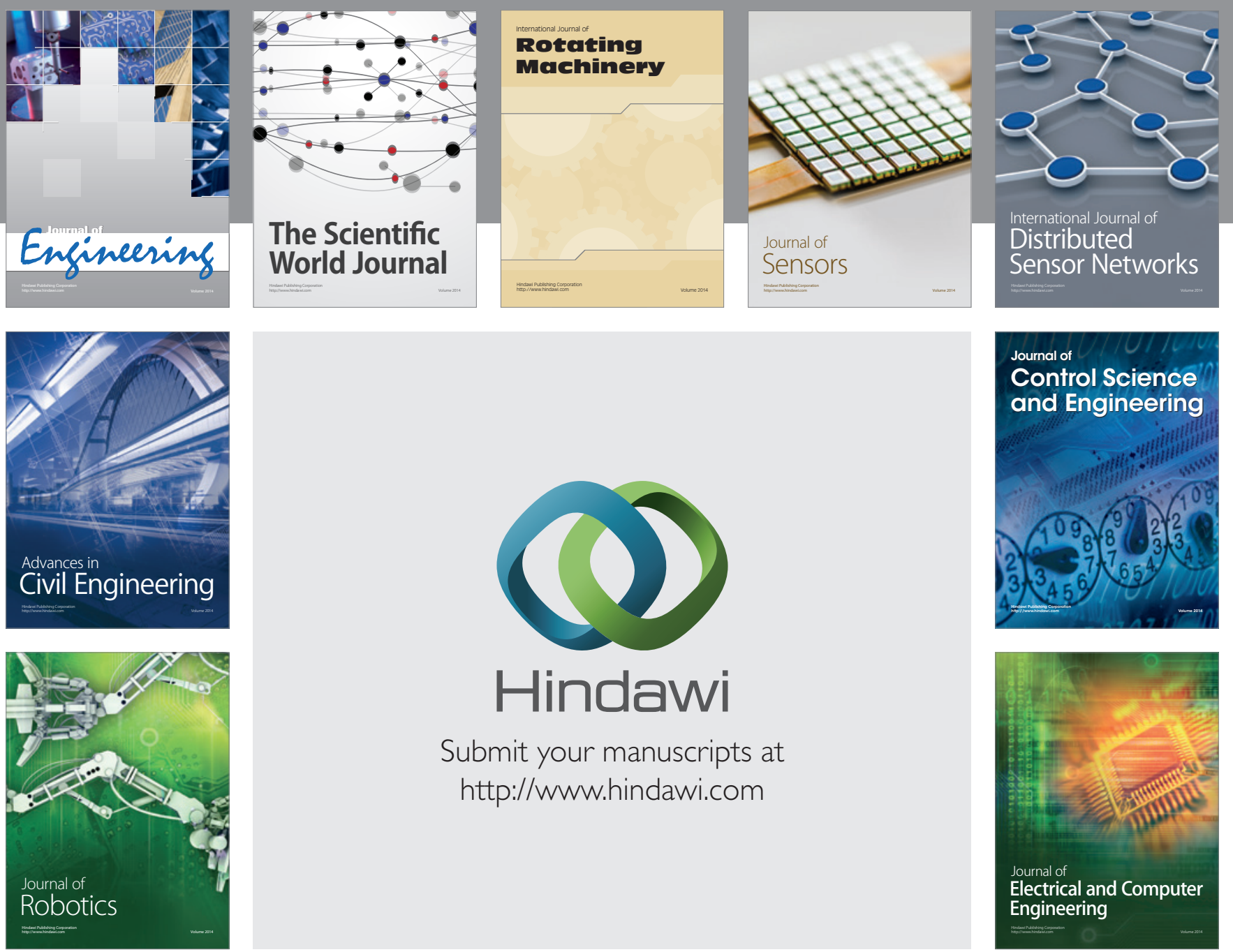

Submit your manuscripts at

http://www.hindawi.com
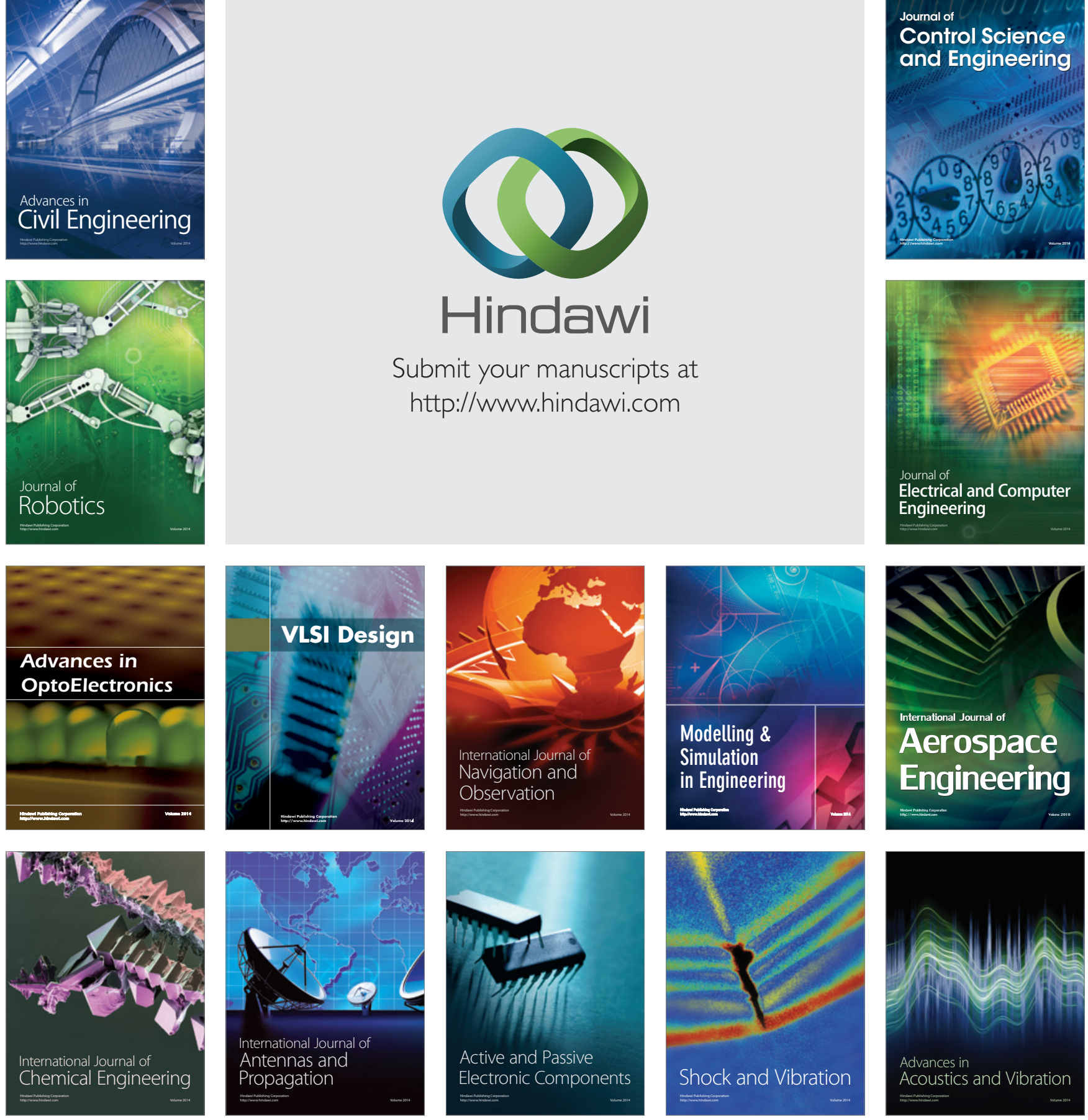